\title{
Correspondence
}

\section{Regional anaesthesia for 12,000 cataract extraction and intraocular lens implantation procedures}

To the Editor:

The interesting article by Hamilton, Gimbel and Strunin (Can J Anaesth 1988; 35: 615-23) concerns the very important subject of how to choose the best method of regional anaesthesia for cataract extractions. The authors compared five methods of anaesthesia, successively applied by the anaesthetist. The results were assessed by the ophthalmologist using an objective scoring system. The five methods of anaesthesia were given in consecutive blocks and not at random. Thus, there may have been a process of learning by the anaesthesist and an influence on the ophthalmologist.

In our hospital we administer $2.5-3.0 \mathrm{ml}$ of a solution containing bupivicaine 0.5 per cent with adrenaline 1:200,000 retrobulbarly into the muscle cone together with $5 \mathrm{ml}$ of this solution into the orbicularis muscle. The authors described that in the five compared methods of anaesthesia they gave a retro- or peribulbar injection of respectively 3-4 ml, 5-7 ml, 7-9 $\mathrm{ml}, 7-9 \mathrm{ml}$ or $10-12 \mathrm{ml}$. Such large injections often produce a bulbar protrusion that impedes a safe operative procedure.

In the assessment of the anaesthetic effects the authors used various relevant criteria but they did not make mention of the ptosis that usually is seen very soon and with the needle still in position. In such cases this symptom indicates that the anaesthesia is well placed and that less solution is needed.

The authors also reported that in seven of the eight patients with brainstem anaesthesia the surgical procedure could be continued. This raises the question of how the diagnosis was made.

Dr. M. Belopavlovic

Dr. L.J. Blanksma

University Hospital Groningen

The Netherlands

\section{REPLY}

Thank you for the opportunity to reply to the letter from Drs. Belopavlovic and Blanksma.

Their correspondence criticizes our article on the grounds that the research method did not meet the criteria of a careful comparison. The paper was entered in the Clinical Reports section of the Journal and at no time purported to be a bona fide research method. The anaesthetist involved $(\mathrm{RCH})$ fully acknowledges that there was a process of learning - which is implied in the text of the article. A large clinical series had been accumulated and it was felt to be worthwhile to report the experiences gained and the problems encountered. The correspondents in their practice use a small volume retrobulbar technique with separate transcutaneous seventh nerve block. In our experience, now exceeding 20,000 regional anaesthetics for cataract extraction, this method does not produce extraocular muscle akinesia in all patients and is associated with intraoperative pain in an unacceptable percentage. On the other hand with a large volume intraorbital technique, akinesia can be obrained in 100 per cent of cases with an incidence of intraoperative breakthrough pain of about 0.1 per cent. Large volume injection often produces bulbar protrusion which makes the use of an orbital decompression device mandatory, as the article states. To embark on surgery without this prerequisite would be foolhardy. In our opinion, large volume injection with subsequent orbital decompression consistently produced surgical conditions superior to those following traditional retrobulbar blocking. Another advantage of a large volume intraorbital technique is the spread of injectate into the periorbital muscle tissue which obviates the need for painful separate transcutaneous seventh nerve blocking.

Our present method is a solely peribulbar, large volume intraorbital technique. We believe that the risk of serious complications associated with attempts to enter within the muscle cone for traditional retrobulbar blocking is such that it should no longer be taught or practised. Papers published in the past 12 months reporting and discussing complications of retrobulbar blocking are listed in the cited references. ${ }^{1-9}$ We fully agree that when there is indication that the anaesthetic agent is well placed, less solution is needed. The capacity and compliance of the tissue spaces in any given orbit may differ greatly from another. Routine digital monitoring of orbital pressure during injection will determine the optimal injectate volume. A "cook-book" approach with predetermined volume will inevitably underdose some orbits and subject others 10 dangerously high pressure.

In the eight patients in whom brainstem anaesthesia occurred severe respiratory depression or arrest was present along with cardiovascular signs of central spread. Three of these have been reported in the literature. ${ }^{10}$ Resuscirative measures were ap. plied for as long as was required until normality and stability returned, after which surgery was performed in all but one. Persistent nausea in this patient rendered her unfit for surgery at that time, but it was successfully completed under regional anaesthesia some days later.

Robert C. Hamilton MB FRCPC Howard V. Gimbel MD FRCSC Leo Strunin MD FFARCS FRCPC

Gimbel Eye Centre

Suite $\mathbf{4 5 0}$

4935 Fortieth Avenue NW

Calgary, Alberta

T3A $2 \mathrm{NI}$ 
REFERENCES

I Jindra $L F$. Blindness following retrobulbar anesthesia for astigmatic keratotomy. Ophthalmic Surg 1989;20: 433-5.

2 Hersch M, Baer G, Dieckert JP, Lambert HM, Shore $J W$. Optic nerve enlargement and central retinal-artery occlusion secondary to retrobulbar anesthesia. Ann Ophthalmol 1989; 21: 195-7.

3 Mercerean DA. Brain-stem anesthesia complicating retrobulbar block. Can J Ophthalmol 1989; 24: 159-61.

4 Morgan $G E$. Post-retrobulbar apnea syndrome. Reg Anesth 1989; 14: 203-5.

5 Hamilton RC. Complications of retrobulbar and peribulbar blocks (Letter to the editor). Reg Anesth 1990; 15: 106.

6 Morgan $G E$. Retrobulbar apnea syndrome: a case for the routine presence of an anesthesiologist (Letter to the editor). Reg Anesth 1990; 15: 107.

7 Rigg JD. James RH. Apnoea after retrobulbar block. Anaesthesia 1989; 44: 26-7.

8 Hamilion RC. Apnoea after retrobulbar block (Letter to the Editor). Anaesthesia 1989; 44: 862-3.

9 Rigg JD, James RH. Apnoea after retrobulbar block. (Letter to the Editor). Anaesthesia 1989; 44: 863.

10 Hamilion $R C$. Brain stem anesthesia following retrobulbar blockade. Anesthesiology 1985; 63: 688-90.

\section{Lambert-Eaton myasthenic syndrome}

The Lambert-Eaton syndrome is thought to be an autoimmune disorder, and immunoglobulin $\mathrm{G}(\mathrm{IgG})$ antibodies from the patients with the syndrome have been shown to act on voltage-dependent calcium channels at nerve terminals to reduce calcium entry and hence acetylcholine release. Patients with the syndrome, however, manifest widespread adrenergic as well as cholinergic dysfunction of the autonomic nervous system, and the $\mathrm{IgG}$ from the patients with the syndrome sometimes fails to reduce quantal content in mice. ${ }^{1}$ Therefore, the pathophysiology associated with the syndrome may not be solely due to the autoantibodies. We had a patient with the syndrome whose muscle response during surgery indicated the possible involvement of factor(s) other than $\mathrm{IgG}$.

A 63-yr-old male with chronic muscle weakness had a lobectomy of the lung performed for adenocarcinoma. The patient was premedicated with atropine, and the trachea was intubated after a small dose of droperidol and fentanyl but without a muscle relaxant. Anaesthesia was maintained with 50 per cent nitrous oxide and epidural mepivacaine (two per cent). Neuromuscular twitch reponse was monitored with an ABM-100 (Datex). The stimulating electrodes were placed over the ulnar nerve, and the recording electrodes over the hypothenar muscles

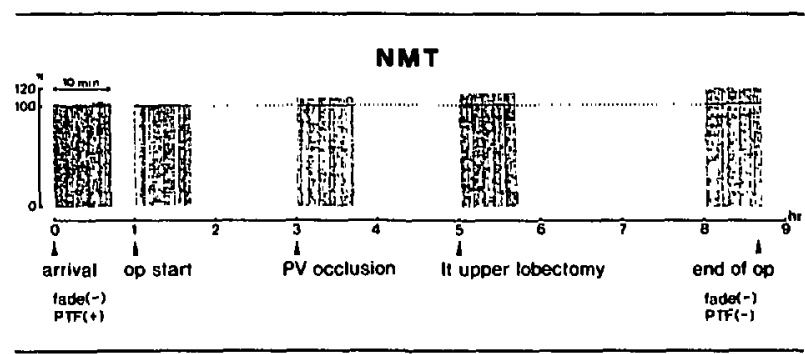

FIGURE $\mathrm{NMT}=$ neuromuscular trend, $\mathrm{PTF}=$ post-tetanic facilitation.

(Figure 1). After ligation of the left upper pulmonary vein, twitch height increased gradually and reached 120 per cent of the preoperative value within $5.5 \mathrm{hr}$. Posttetanci facilitation, which was observed at the start of the operation, disappeared at the end of the operation. Although the skin temperature was not monitored, the rectal temperature was 37.6 and $38.2^{\circ} \mathrm{C}$ at the times of ligation of the pulmonary vein and at the end of operation, respectively. Electrolyte imbalance was not observed during the operation. This immediate improvement of the reduced muscle response does not seem to be explained by the involvement of $\operatorname{IgG}$ as a single aetiology, because IgG has a half-life of about 21 days. A factor or factors other than IgG seem to be involved in the myasthenic syndrome observed in our patient. We suggest that this (or these) factor(s) is secreted from the tumour, affects neuromuscular transmission, and has a relatively short half-life. Our patient may be unusual, because the histology of the tumour showed it to be an adenocarcinoma and not small-cell carcinoma.

\section{Kazuo Irita MD PhD}

Michiko Satoh MD

Takashi Akata MD

Takashi Yano MD

Shosuke Takahashi MD PhD

Junichi Yoshitake MD PhD

Department of Anesthesiology and Critical Care Medicine

Faculty of Medicine, Kyushu University

3-1-1 Maidashi, Higashi-ku, Fukuoka, 812 Japan

\section{REFERENCE}

1 Wray DW, Peers $C$, Lang B, Lande S, Davis JN. Interference with calcium channels by Lambert Eaton myasthenic syndrome antibody. Ann NY Acad Sci 1987; 505: $368-76$ 